\title{
Pericarditis in a Child with an Intrapericardial Bronchogenic Cyst
}

\author{
Tim Attmann ${ }^{1}$ Christina Grothusen ${ }^{1}$ Carsten Rickers ${ }^{2}$ Peter Dütschke ${ }^{3}$ Hans-Heiner Kramer ${ }^{2}$ \\ Jens Scheewe ${ }^{1}$
}

${ }^{1}$ Department of Cardiovascular Surgery, University Hospital Schleswig-Holstein, Campus Kiel, Kiel, Germany

2 Department of Congenital Heart Disease and Pediatric Cardiology, University Hospital of Schleswig-Holstein, Campus Kiel, Kiel, Germany

${ }^{3}$ Department of Anaesthesiology, University Hospital SchleswigHolstein, Campus Kiel, Kiel, Germany

Thorac Cardiovasc Surg Rep 2016;5:47-49.

\author{
Address for correspondence Christina Grothusen, MD, Department of \\ Cardiovascular Surgery, University Hospital Schleswig-Holstein, \\ Campus Kiel, Arnold-Heller Straße 3, Kiel 24105, Germany \\ (e-mail: christinagrothusen@yahoo.de).
}

\begin{abstract}
Keywords

- pericarditis

- cardiac anatomy

- pathologic anatomy

- mediastinal tumor

Background An intrapericardial bronchogenic cyst (IBC) is a rare congenital malformation seemingly asymptomatic in most subjects.

Case Description A 9-year-old boy presented with persistent chest pain and fever. Imaging revealed a large pericardial effusion with a tumor located at the left atrial appendage extending behind the great vessels. Mass rupture with scattered mucoid debris was found intra-operatively. The tumor was excised and the IBC was confirmed by immunohistochemistry. A postcardiotomy syndrome occurred four weeks post-operative. It was treated conservatively. Since then, follow-up visits have remained uneventful.

Conclusion IBCs are a scarce, but serious cause of persistent inflammation in children.
\end{abstract}

\section{Introduction}

Bronchogenic cysts are a rare congenital malformation occurring due to abnormal budding of the tracheobronchial tree and the embryonic foregut. ${ }^{1}$ While these masses underlie most congenital mediastinal cysts, the intra-pericardial location has been described as being very rare. However, the true incidence of these cysts is unknown, as most patients are believed to remain asymptomatic. However, depending on the location, bronchogenic cysts may cause a variety of symptoms, including infection and compression. In addition, malignant transformation has been described. ${ }^{2}$

\section{Case Description}

A 9-year-old boy suffered from influenza-like symptoms with progressive chest pain for two weeks. Chest X-ray showed a broadened heart shadow so the patient was admitted to our institution. Pathologic laboratory findings revealed elevated values for $\mathrm{C}$-reactive protein and white blood cell count (neutrophilia). Transthoracic echocardiography demonstrated a large circular pericardial effusion. A cystic mass of $6 \times 6 \times 4 \mathrm{~cm}$ in size was seen in the vicinity of the left atrium extending behind the great vessels. Thoracic magnetic resonance imaging (MRI) scan (-Fig. 1) confirmed an extensive, smooth, cystoid tumor without signs of infiltrative growth. Vascularization solely concerned the capsule of the mass. A diagnostic coronary angiography was performed to rule out any connection between the tumor and the coronary vasculature. Indication for surgical excision was made. Due to the cyst location, we performed a sternotomy in case cardio-pulmonary bypass (CPB) was needed. Intra-operatively, the pericardium was thickened. The epicardium proved to be thickened as well and seemed inflamed. We also found extensive mucoid debris indicating that rupture had already occurred ( - Fig. 2A, arrow). In addition, strong adhesions of the cyst
License terms

Stuttgart · New York

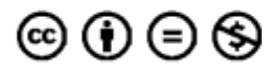

November 3, 2015 accepted after revision November 30, 2015 published online February 12, 2016
DOI http://dx.doi.org/ 10.1055/s-0035-1571139. ISSN 2194-7635. 


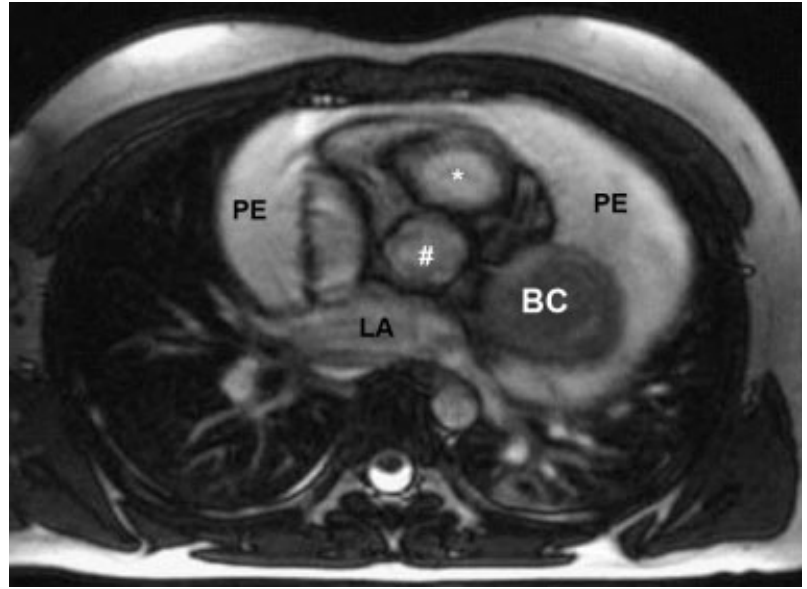

Fig. 1 Magnetic resonance tomography demonstrating the bronchogenic cyst (BC) and the large pericardial effusion (PE). LA, Left atrium. *Right ventricular outflow tract. ${ }^{\# A s c e n d i n g ~ a o r t a, . ~}$

to the pericardium and to the left atrial appendage were present. Luxation of the heart was not possible without inducing hemodynamic instability. Therefore, СРB was established by cannulating the ascending aorta and the right atrium. During dissection of the adhesions, the tumor ( - Fig. 2B, arrow) was opened unintentionally, leading to release of mucoid secretion ( $\mathbf{- F i g} . \mathbf{2 C}, \mathbf{2 D}$ ). The mass was completely removed. The microbial analysis remained inconspicuous. The immunohistochemical staining showed cystic material lined with respiratory endothelium and small islets of cartilage confirming a bronchogenic cyst with signs of a purulent, chronic, and granulating inflammation. The patient underwent an uneventful recovery. However, the child was re-admitted four weeks after the initial procedure with fever, elevated C-reactive protein and leucocytosis caused by neutrophilia. Sonography revealed a novel pericardial and pleural effusion that was interpreted as a postcardiotomy syndrome and treated with Ibuprofen, a non-steroidal anti-inflammatory drug (NSAID). The pleural effusion was successfully drained. Microbiological analysis of several blood samples and the serous pleural fluid remained inconspicuous. The pericardial effusion, fever, and elevated inflammatory markers dissolved spontaneously under NSAID treatment. The child could be discharged after 8 days. Half-yearly ambulatory visits have remained uneventful ever since.

\section{Discussion}

We hereby present a case of an intrapericardial bronchogenic cyst (IBC) in a child resulting in a large pericardial effusion and a systemic inflammatory response. Those symptoms were most likely caused by rupture of the cyst, given that the epicardium was covered with mucoid debris and both epiand pericardium showed inflammatory remodelling intraoperatively. Pericarditis as well as rupture along with fever and chest pain have already been described in context with $\mathrm{IBC}^{3,4}$ However, we did not find any report about this combination in the child.

While other cyst locations enable minimally-invasive procedures, we chose to perform a sternotomy. Given the fact that we experienced hemodynamic instability while trying to expose the cyst in addition to the circumstance that the rupture of the cyst had led to strong adhesions with the surrounding tissue, we feel that sternotomy as well as use of endocervical curettage (CPB) was justified in this case. Furthermore, lateral thoracotomies may result in one-sided growth retardation and therefore, should be done with caution in children. ${ }^{5}$

Postcardiotomy syndromes occur in approximately $10 \%$ of patients after pediatric cardiac surgery. ${ }^{6}$ Although a potential benefit by treatment of NSAD has not been proven so far, we observed full recovery after administration of Ibuprofen. We did not detect any signs of IBC recurrence during the regularly performed ambulatory visits. In summary, an IBC represents a
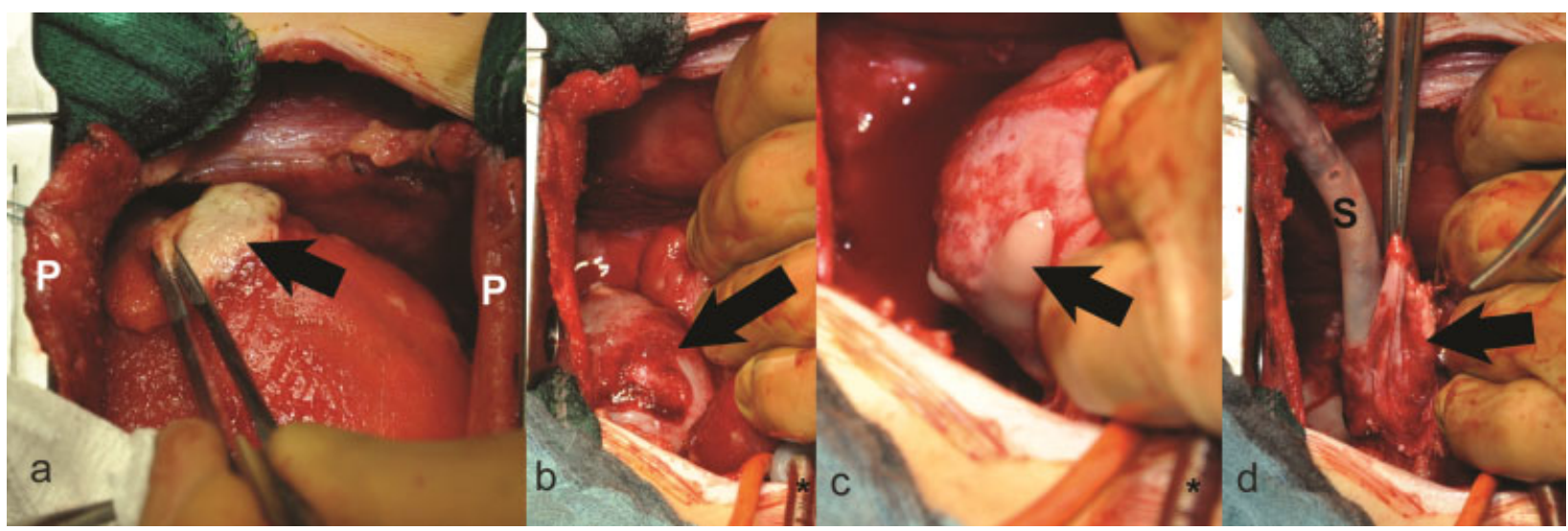

Fig. 2 (a) Thickened pericardium (P) and mucoid debris covering the epicardium (black arrow). (b) with the heart pulled to the right, the tumor (black arrow) is exposed. *Arterial cannula (c) mucoid secretion (black arrow) after accidental opening of the tumor. *Arterial cannula (d) Forceps holding the wall of the bronchogenic cyst (black arrow) after evacuation by suction (S). 
rare but possible cause of systemic inflammation in children. Given the severe symptoms caused by the IBC in this case, we recommend that surgical excision should be performed as soon as the suspected diagnosis of an IBC has been made.

\section{References}

1 Wright CD. Mediastinal tumors and cysts in the pediatric population. Thorac Surg Clin 2009;19(1):47-61, vi vi.

2 Okada Y, Mori H, Maeda T, Obashi A, Itoh Y, Doi K. Congenital mediastinal bronchogenic cyst with malignant transformation: an autopsy report. Pathol Int 1996;46(8):594-600
3 Sanjeeva NCG, Sarkar A, Ahmed I, Patil S. Large Bronchogenic cyst Masquerading as Pericardial cyst and Causing Congenital lobar Emphysema. Journal of Cardiovascular Disease Research. 2015; 6:97-99

$4 \mathrm{Li}$ Z, Wang X, Yang E, Gao K, Huang L. Gigantic intrapericardial bronchogenic cyst. Neth Heart J 2011;19(12): 532-533

5 Panda SS, Agarwala S, Bhatnagar V, Kabra SK, Jayaswal A, Bhalla AS. A survey of musculoskeletal and aesthetic abnormalities after thoracotomy in pediatric patients. J Indian Assoc Pediatr Surg 2013;18(4):136-142

6 Dalili M, Zamani H, Aarabi-Moghaddam M. Pericardial effusion after pediatric cardiac surgeries: a single center observation. Rev Cardiovasc Med 2012;1(1):28-32 